\title{
Integrated Ventricular Mechanics in "Healthy" Heart Transplant Patients
}

\author{
Luca Baldini, MD*; Giuseppe Limongelli, MD, PhD, FESC*; Cristiano Amarelli**, \\ MD; Lucia Riegler, MD*; Rita Gravino, MD*; Alessandra Rea, MD*; Gemma Salerno, \\ MD*; Daniele Masarone, MD*; Antonello D'Andrea, MD, PhD, FESC*; Giovanni Di \\ Salvo, MD, PhD, MMSc, FESC*; Raffaele Calabro', MD*; Elliott PM, MD***; \\ Giuseppe Pacileo, MD*. \\ *Cardiomyopathies and Heart Failure Unit, A.O. dei Colli, Naples, Italy. \\ ** Heart Transplantation Unit, A.O. dei Colli, Naples, Italy. \\ *** The Heart Hospital, 16-18 Westmoreland Street, London W1G 8PH
}

\begin{abstract}
Although recently studies using Speckle Tracking Echocardiography (STE) on heart transplant (HTX) recipients have been published, an integrated assessment of myocardial properties is lacking. Thus our aim was to perform, by STE, an integrated study of ventricular mechanics in "healthy" HTX recipients. Standard echo-Doppler and STE study were performed in 50 HTX patients (age 50.4 $\pm 7.8, M / F=37 / 13$, follow-up $7.6 \pm 5.3$ years) without symptoms (NYHA I) and with preserved ejection fraction (EF) (HTX Group). As controls 35 age-, sex-and BSA-matched normal subjects were studied (CTRL Group).

Compared to CTRL group our HTX recipients showed: a) impaired left ventricular (LV) longitudinal, circumferential and radial Strain values ( $p<0.01)$ with a lesser degree of reduction at the apical segments; $b$ ) normal apical longitudinal, circumferential and radial Strain Rate values with reduction in the remaining segments; c) preservation of LV apical rotation and basal impairment ( $p<0.0001)$ with normal LV twist; d) delayed untwisting due to prolonged twist $(p<0.0001)$ and thus prolongation of isovolumic relaxation time $(p<0.01)$ in presence of normal E/E' ratio; e) reduced right ventricular TAPSE, $S^{\prime}(p<0.0001)$ and longitudinal Strain and $S R(p<0.0001)$.

"Healthy" HTX patients, even in presence of impaired global biventricular myocardial deformation, show normal amplitude of $L V$ twist and in turn EF. In addition, probably due to regional differences in wall stress, a $L V$ base-apex gradient of these subclinical systolic dysfunction is detectable. Finally an early impaired LV diastolic filling due to delayed untwisting related to prolonged twist is also evident.
\end{abstract}

Keywords: Heart Transplantation • Echocardiography • Heart function • Speckle Tracking Echocardiography• Pathophysiology.

\section{INTRODUCTION}

Heart transplant (HTX) patients have an high risk for many heart complications due to chronic immunosuppressive therapy, rejection and allograft vasculopathy [1-2]. For this reason an accurate evaluation of cardiac function is needed and echocardiography represents a key tool in the management of heart transplant recipients. However, although standard 2-dimensional and Doppler echocardiography show some limitations, such as subjective visual assessment of heart function and measurement of thickening only on the radial plane missing the other myocardial deformations properties, new echocardiographic techniques could overcome them [3].Particularly, two-dimensional Speckle Tracking Echocardiography (STE) allows us to evaluate longitudinal, circumferential and radial myocardial deformation as well as rotation, twist and untwist properties in different settings, it is able to unmask changes in left ventricular (LV) function at an earlier sub-clinical stage [4-9] and has an important prognostic role in detecting subgroups at risk of major events [10].

Despite recent publications revealed that STE-derived strain analysis is feasible and practical in HTX subjects [11-15], an integrated assessment of myocardial properties is lacking. Thus, our aim was to perform, by STE, an integrated study of ventricular mechanics in "healthy" HTX recipients, in order to acquire new pathophysiological insights on the mechanisms underlying myocardial dysfunction in these particular setting of patients. 


\section{Materials AND Methods}

\subsection{Patients Selection}

Between January 2011 and October 2012, 154 consecutive pts with heart transplantation were assessed at our "Cardiomyopathies and Heart Failure Unit".

Inclusion criteria to the study were: a) preserved ejection fraction (EF > 50\%); b) good clinical conditions (NYHA class I); c) heart transplantation performed at least 1 year before; d) normal myocardial perfusion assessed by scintigraphy in the last twelve months.

Exclusion criteria to the study were: a) inadequate echocardiographic resolution; b) patients with implantable cardioverter-defibrillators; c) history of histologic evidence of severe allograft rejection (Grade 2R or higher) based on the 2004 revised grading system of the International Society for Heart and Lung Transplantation (ISHLT) [16]; d) comorbilities such as diabetes mellitus, arterial hypertension refractory to medical therapy, coronary artery disease, atrial fibrillation during the study evaluation, significant valvular disease.

Among all the patients, 104 were excluded from the study (81 owing to the presence of exclusion criteria and 23 to inadequate echocardiographic images). The remaining 50 were enrolled for the study (HTX Group). In addition we studied 35 age-, sex- and BSA- matched normal subjects (CTRL Group). None of the control subjects had cardiovascular structural or functional abnormalities or received any medication.

\subsection{Standard Echo-Doppler Study}

Standard echo-Doppler was performed using a Vivid 7 ultrasound system (GE Vingmed Ultrasound AS, Horten, Norway). Cine-loops were recorded on DVDs for offline analysis (EchoPAC PC 6.0.0, GE Medical Systems). All the measurements were analyzed by two experienced readers, taking the average of 3 cardiac cycles.

LV diameter and wall thickness were measured according to the criteria of the American Society of Echocardiography [17]. Left atrium (LA) volume was determined by the biplane-area-length method [18]. In addition, LV EF was calculated by Simpson biplane method [19].

As measures of global LV diastolic function mitral peak velocities at the early (peak E) and late (peak A) diastole, their ratio, deceleration time of the $\mathrm{E}$ wave and isovolumic relaxation time (IVRT) were assessed by pulsed-Doppler with the sample volume placed at the mitral valve leaflet tips and at the aortic outflow [20].

By pulsed-wave tissue Doppler, peak early diastolic velocity of the mitral annulus was measured (E') and $\mathrm{E} / \mathrm{E}$ ' ratio was calculated [21].

Finally, right ventricular (RV) systolic function was assessed in the apical four-chamber view by measuring lateral tricuspid annular excursion (TAPSE), a previously validated parameter for assessment of ventricular function [22] and by the systolic peak velocity of the pulsed-wave tissue Doppler recordings from the basal segment of the RV free wall, adjacent to the tricuspid annulus (S') [23].

\subsection{Speckle Tracking Echocardiography Study}

For the STE study the second-harmonic B-mode images of apical (4-chamber, 2-chamber. 3-chamber) and short axis (at the mitral valve, mid LV and apical level) views were obtained. The frame rate was $79 \pm 14 \mathrm{frame} / \mathrm{s}$. The endocardial border was manually traced at the end-systolic frame and a speckle tracking region of interest was automatically selected. The width of the region of interest was adjusted as necessary to accommodate the total thickness of the ventricular wall. The computer automatically tracked stable objects in each frame using the sum of absolute differences algorithm. After these steps, the workstation computed and generated strain curves.

In order to assess LV strain and strain rate (SR) we utilized apical views (4-, 2- and 3- chambers) for the longitudinal and parasternal short axis views (basal, mid and apical level) for the circumferential and radial myocardial deformations as well as LV rotation functions (rotation, rotation rate, twist and twist rate)

The average between all regional values (18 segments) for each myocardial deformation were considered to assess global longitudinal, circumferential and radial strain and SR. 
On the other hand for RV longitudinal strain and strain rate (SR) apical 4-chamber view comprehensive of 6 segments (septum and lateral wall) was considered. Longitudinal and circumferential strain and SR peak values were defined as the maximum negative values of the curves from the apical and short axis views, respectively. Radial strain and SR peak values were defined as the maximum positive values of the curves from the short axis views.

The peak values of basal rotation (basal rotation and rotation rate) were defined as the maximum negative values of the curves from the short axis view at the mitral valve level.

The peak values of apical rotation (apical rotation and rotation rate) were defined as the maximum positive values of the curves from the short axis view at the apical level. Every view considered was divided in 6 segments which gave 6 different values : the mean value of them was considered.

LV twist was defined as the difference between the mean values of the peak rotation at the apical and at the mitral valve level (twist = mean peak apical rotation - mean peak basal rotation).

Similarly LV twist rate was defined as the difference between the mean values of the peak rotation rate at the apical and at the mitral valve level (twist rate = mean peak apical rotation rate - mean peak basal rotation rate). The untwisting onset was expressed as a percentage of systolic duration (the ratio between the time to peak twist, i.e onset of untwisting, and the duration of systole until the aortic valve closure) by the use of cardiac cycles with matched RR intervals (time to peak twist/systolic time). This normalization for systolic duration was made to overcome the heart rate dependence, as previously described [24]. The studies were analyzed off-line by a second blinded observer for 20 patients, corresponding to 840 segments.

Intraobserver variability was calculated by the average difference between the 42 measurements reanalyzed. Interobserver variability was calculated as the absolute difference divided by the average of the two observations for all parameters.

\subsection{Ethics}

The study was approved by the local research ethics committee and informed written consent was obtained from all participants.

\subsection{Statistical Analysis}

Data are expressed as mean \pm standard deviation (SD). Clinical and demographic characteristics were compared using the Mann-Whitney $U$ test for continuous variables and the $\chi^{2}$ test for categorical variables.

The correlation structure set of metric parameters was inspected using Pearson's product-moment correlation coefficient.

A P value $<0.05$ was considered statistically significant. StatView (SAS Institute Inc, Cary, NC) was used for all analyses.

\section{RESULTS AND DISCUSSION}

\subsection{Characteristics Of The Study Population}

The general characteristics of the studied groups are shown in Table 1. The mean time post-heart transplant was $7.6 \pm 5.3$ years. Compared to CTRL Group, HTX Group was comparable for age, sex, BSA and blood pressure, with a significant higher heart rate (HTX $87 \pm 7.3 \mathrm{bpm}$ vs CTRL Group $75.3 \pm 9.4 \mathrm{bpm} ; p$ value <0.0001), due to heart denervation.

At the time of the study thirty-four out of fifty HTX patients were under cardiac medical therapy (only on ace-inhibitors). Patients did not withdraw therapy before the echocardiographic evaluation, according to the rules of our institutions' research ethical committees.

TABLE 1. General characteristics of the studied groups.

\begin{tabular}{|l|c|c|c|}
\hline & CTRL & HTX Group & $\boldsymbol{p}$ value \\
\hline $\mathbf{N}^{\circ}$ & 35 & 50 & \\
\hline Sex $(\mathbf{M} / \mathbf{F})$ & $23 / 12$ & $37 / 13$ & NS \\
\hline Age $(\mathbf{y r s})$ & $48.5 \pm 5.4$ & $50.4 \pm 7.8$ & NS \\
\hline BSA $\left(\mathbf{m}^{2}\right)$ & $1.75 \pm 0.3$ & $1.81 \pm 0.4$ & NS \\
\hline SBP $(\mathbf{m m H g})$ & $118.5 \pm 8.1$ & $115.4 \pm 12.6$ & NS \\
\hline DBP $(\mathbf{m m H g})$ & $75.8 \pm 6.3$ & $73.9 \pm 5.5$ & NS \\
\hline HR (bpm) & $75.3 \pm 9.4$ & $87 \pm 7.3^{*}$ & $<0.0001$ \\
\hline
\end{tabular}


Legend: $\mathrm{BSA}=$ body surface area; $\mathrm{CTRL}=$ control group; $\mathrm{DBP}=$ diastolic blood pressure; $\mathrm{SBP}=$ systolic blood pressure; HR = heart rate; HTX = Heart Transplantation.

\subsection{Standard Echo-Doppler analysis}

All standard echocardiographic data are shown in Table 2.

Compared to CTRL, HTX showed left atrial enlargement (LAVI $37.9 \pm 5.1 \mathrm{ml} / \mathrm{m} 2$ vs $26.4 \pm 4.1$ $\mathrm{ml} / \mathrm{m} 2, \mathrm{p}<0.01$ ) and a decreased longitudinal excursion of the right ventricle free wall (TAPSE 14.42 \pm 4.54 vs $19.5 \pm 2.1 \mathrm{~mm} ; \mathrm{p}<0.0001)$ as well as systolic velocity peak of the pulsed-wave tissue Doppler (RV free wall S' $9.52 \pm 2.2 \mathrm{~cm} / \mathrm{s}$ vs $13.5 \pm 1.9 \mathrm{~cm} / \mathrm{s} ; \mathrm{p}<0.0001$ ).

In addition, HTX patients showed an increased IVRT $(81.52 \pm 9.19 \mathrm{msec}$ vs $71.8 \pm 5.4 \mathrm{msec} ; \mathrm{p}$ $<0.0001$ ), suggestive of an early impairment of diastolic function.

TABLE 2. Standard Echocardiographic values of the studied groups.

\begin{tabular}{|l|c|c|c|}
\hline & CTRL & HTX Group & p value \\
\hline $\mathbf{N}^{\circ}$ & 35 & 50 & NS \\
\hline LVDed (mm) & $48.1 \pm 2.3$ & $47.36 \pm 5.27$ & NS \\
\hline IVSed (mm) & $9.7 \pm 1.9$ & $10.1 \pm 1.1$ & NS \\
\hline PWed $(\mathbf{m m})$ & $9.5 \pm 1.7$ & $9.8 \pm 1.1$ & NS \\
\hline LAVI $\left(\mathbf{m l} / \mathbf{m}^{2}\right)$ & $26.4 \pm 4.1$ & $37.9 \pm 5.1$ & NS \\
\hline EF $(\%)$ & $59.4 \pm 5.5$ & $58.44 \pm 4.34$ & $<0.01$ \\
\hline E/A & $1.65 \pm 0.2$ & $1.48 \pm 0.7$ & NS \\
\hline DecTime (msec) & $168.4 \pm 29.1$ & $184.5 \pm 25.4$ & $<0.0001$ \\
\hline E/E' & $5.3 \pm 1.8$ & $5.65 \pm 2.09$ & $<0.0001$ \\
\hline IVRT (msec) & $71.8 \pm 5.4$ & $81.52 \pm 9.19$ & $<0.0001$ \\
\hline TAPSE (mm) & $19.5 \pm 2.1$ & $14.42 \pm 4.54$ & \\
\hline RV free wall S' (cm/s) & $13.5 \pm 1.9$ & $9.52 \pm 2.2$ & \\
\hline
\end{tabular}

Legend:Dec Time=deceleration time; $\mathrm{EF}=$ ejection fraction; IVRT = isovolumic relaxation time;IVSed = end-diastolic interventricular septum; LAVI =left atrium volume indexed; LVEDed = end-diastolic left ventriculardiameter; PWed = end-diastolic posterior wall; RV = right ventricular;

TAPSE $=$ tricuspid annular plane systolic excursion. For HTX see table 1.

\subsection{Speckle Tracking Echocardiography Study}

All STI analysis data are shown in Table 3, Table 4 and Table 5. Compared to CTRL, HTX patients showed significantly lower values of LV global longitudinal, circumferential and radial, and RV longitudinal myocardial deformation (Strain and SR).

TABLE 3. Speckle Tracking Analysis of the studied groups (Global S and SR).

\begin{tabular}{|c|c|c|c|}
\hline & CTRL & HTX Group & p value \\
\hline $\mathbf{N}^{\circ}$ & 35 & 50 & \\
\hline LV Long Strain (\%) & $-21.5 \pm 1.5$ & $-17.56 \pm 3.2$ & $<0.0001$ \\
\hline LV Long SR $\left(\mathrm{s}^{-1}\right)$ & $-1.46 \pm 0.21$ & $-1.29 \pm 0.28$ & $<0.005$ \\
\hline LV Circ Strain (\%) & $-22.5 \pm 2.9$ & $-18.6 \pm 5.1$ & $<0.0001$ \\
\hline LV Circ SR $\left(\mathrm{s}^{-1}\right)$ & $-1.84 \pm 0.25$ & $-1.5 \pm 0.39$ & $<0.0001$ \\
\hline LV Rad Strain (\%) & $45.1 \pm 9.6$ & $37.02 \pm 13.9$ & $<0.005$ \\
\hline LV Rad SR $\left(\mathrm{s}^{-1}\right)$ & $2.17 \pm 0.24$ & $1.91 \pm 0.57$ & $<0.05$ \\
\hline LV TWIST $\left(^{\circ}\right)$ & $13.91 \pm 2.8$ & $12.64 \pm 3.9$ & NS \\
\hline LV TWIST Rate $\left({ }^{\circ} / \mathrm{s}\right)$ & $137.6 \pm 14.3$ & $128.3 \pm 12.4$ & $<0.005$ \\
\hline LV Untwist Onset (\%) & $84.7 \pm 11.5$ & $100.5 \pm 11.09$ & $<0.0001$ \\
\hline RV Long Strain (\%) & $-23.2 \pm 1.5$ & $-19.3 \pm 1.2$ & $<0.0001$ \\
\hline RV Long SR $\left(\mathrm{s}^{-1}\right)$ & $-1.51 \pm 0.3$ & $-1.25 \pm 0.2$ & $<0.0001$ \\
\hline
\end{tabular}

Legend: Ap = apical; Bas = basal; Circ = circumferential; Long = longitudinal; $\mathrm{LV}=$ left ventricular $\mathrm{Rad}=$ radial; RotRate $=$ Rotation Rate $\mathrm{RV}=$ rightventricular; $\mathrm{SR}=$ Strain Rate. For HTX see table 1. 
Integrated Ventricular Mechanics in "Healthy" Heart Transplant Patients

TABLE 4. Speckle Tracking Analysis of the studied groups: comparison between LV layers.

\begin{tabular}{|c|c|c|c|}
\hline & CTRL & HTX Group & p value \\
\hline $\mathbf{N}^{\circ}$ & 35 & 50 & \\
\hline LV Long Strain bas(\%) & $-21.08 \pm 2.2$ & $-16.78 \pm 3.6$ & $<0.0001$ \\
\hline LV Long Strain mid (\%) & $-20.62 \pm 1.5$ & $-16.79 \pm 3.5$ & $<0.0001$ \\
\hline LV Long Strain ap (\%) & $-21.7 \pm 1.8$ & $-19.8 \pm 4.5$ & $<0.05$ \\
\hline LV Long SR bas $\left(\mathrm{s}^{-1}\right)$ & $-1.5 \pm 0.2$ & $-1.3 \pm 0.3$ & $<0.001$ \\
\hline LV Long SR mid (\%) & $-1.28 \pm 0.2$ & $-1.16 \pm 0.3$ & $<0.05$ \\
\hline LV Long SR ap $\left(\mathrm{s}^{-1}\right)$ & $-1.42 \pm 0.2$ & $-1.43 \pm 0.3$ & NS \\
\hline LV Circ Strain bas $(\%)$ & $-22.9 \pm 3.9$ & $-17.03 \pm 4.3$ & $<0.0001$ \\
\hline LV Circ Strain mid (\%) & $-21.81 \pm 3.5$ & $-17.79 \pm 3.6$ & $<0.0001$ \\
\hline LV Circ Strain ap (\%) & $-22.19 \pm 5.5$ & $-19.8 \pm 4.5$ & $<0.05$ \\
\hline LV Circ SR bas $\left(\mathrm{s}^{-1}\right)$ & $-1.9 \pm 0.4$ & $-1.41 \pm 0.4$ & $<0.0001$ \\
\hline LV Circ SR mid (\%) & $-1.75 \pm 0.3$ & $-1.32 \pm 0.3$ & $<0.0001$ \\
\hline LV Circ SR ap $\left(\mathrm{s}^{-1}\right)$ & $-1.8 \pm 0.4$ & $-1.65 \pm 0.4$ & NS \\
\hline LV Rad Strain bas (\%) & $44.5 \pm 12.2$ & $34.7 \pm 17.1$ & $<0.005$ \\
\hline LV Rad Strain mid (\%) & $45.31 \pm 16.03$ & $35.13 \pm 18.8$ & $<0.05$ \\
\hline LV Rad Strain ap (\%) & $45.6 \pm 17.4$ & $35.8 \pm 19.3$ & $<0.05$ \\
\hline LV Rad SR bas $\left(\mathrm{s}^{-1}\right)$ & $2.2 \pm 0.5$ & $1.8 \pm 0.5$ & $<0.01$ \\
\hline LV Rad SR mid (\%) & $2.2 \pm 0.8$ & $1.8 \pm 0.6$ & $<0.05$ \\
\hline LV Rad SR ap $\left(\mathrm{s}^{-1}\right)$ & $2.1 \pm 0.7$ & $1.9 \pm 0.8$ & NS \\
\hline LV Bas Rotation $\left(^{\circ}\right)$ & $-7.26 \pm 1.45$ & $-5.09 \pm 2.15$ & $<0.0001$ \\
\hline LV Ap Rotation $\left(^{\circ}\right)$ & $7.13 \pm 2.31$ & $7.61 \pm 3.4$ & NS \\
\hline LV Bas RotRate ( $\left.{ }^{\circ} / \mathrm{s}\right)$ & $-75.23 \pm 15.2$ & $-51.6 \pm 20.01$ & $<0.0001$ \\
\hline LV Ap RotRate $(\% / s)$ & $64.7 \pm 15.7$ & $69.55 \pm 25.7$ & NS \\
\hline LV Bas Untwist Onset (\%) & $85.3 \pm 12.2$ & $106.4 \pm 20.3$ & $<0.0001$ \\
\hline LV Ap Untwist Onset (\%) & $84.9 \pm 13.7$ & $89.5 \pm 12.09$ & NS \\
\hline
\end{tabular}

Legend: $A p=$ apical; Bas = basal; Circ = circumferential; Long = longitudinal; Rad =radial; RotRate = Rotation Rate; $S R=$ Strain Rate.For HTX and $L V$ see table 1 and 3.

TABLE 5. Speckle Tracking Analysis of RV in the studied groups.

\begin{tabular}{|c|c|c|c|}
\hline & CTRL & HTX Group & p value \\
\hline $\mathbf{N}^{\circ}$ & 35 & 50 & \\
\hline RV Long Strain bas (\%) & $-25.6 \pm 7.1$ & $-20.5 \pm 3.4$ & $<0.0001$ \\
\hline RV Long Strain mid (\%) & $-22.4 \pm 4.3$ & $-20.1 \pm 2.8$ & $<0.005$ \\
\hline RV Long Strain ap (\%) & $-20.3 \pm 2.2$ & $-18.6 \pm 2.5$ & $<0.005$ \\
\hline RV Long SR bas $\left(\mathrm{s}^{-1}\right)$ & $-1.65 \pm 0.2$ & $-1.38 \pm 0.4$ & $<0.0005$ \\
\hline RV Long SR mid $\left(\mathrm{s}^{-1}\right)$ & $-1.5 \pm 0.4$ & $-1.23 \pm 0.2$ & $<0.0001$ \\
\hline RV Long SR ap $\left(\mathrm{s}^{-1}\right)$ & $-1.43 \pm 0.6$ & $-1.14 \pm 0.3$ & $<0.0005$ \\
\hline
\end{tabular}

Legend: Ap = apical; Bas = basal; Long = longitudinal; $\mathrm{SR}=$ Strain Rate.For HTX and RV see table 1 and 3 .

Of interest, when considering LV segmental values, in contrast to widespread reduction in the remaining segments, at the apex HTX pts showed a lesser but still significant reduction of Strain values $(\mathrm{p}<0.05)$ and normal longitudinal, circumferential and radial Strain Rate values.

In addition, compared to CTRL, HTX pts had a lower basal rotation with a normal amplitude of apical rotation and then twist $\left(\right.$ HTX Group $=$ Basal Rotation $-5.09^{\circ} \pm 2.15^{\circ}$, Apical Rotation $7.61^{\circ} \pm 3.4^{\circ}$ Global Twist $12.64^{\circ} \pm 3.9^{\circ}$; CTRL Group $=$ Basal Rotation $-7.26^{\circ} \pm 1.45^{\circ}$, Apical Rotation $7.13^{\circ} \pm$ $2.31^{\circ}$ Global Twist $13.91^{\circ} \pm 2.8^{\circ}$; p values <0.0001, p NS, p NS, respectively) (Figure 1,2). 


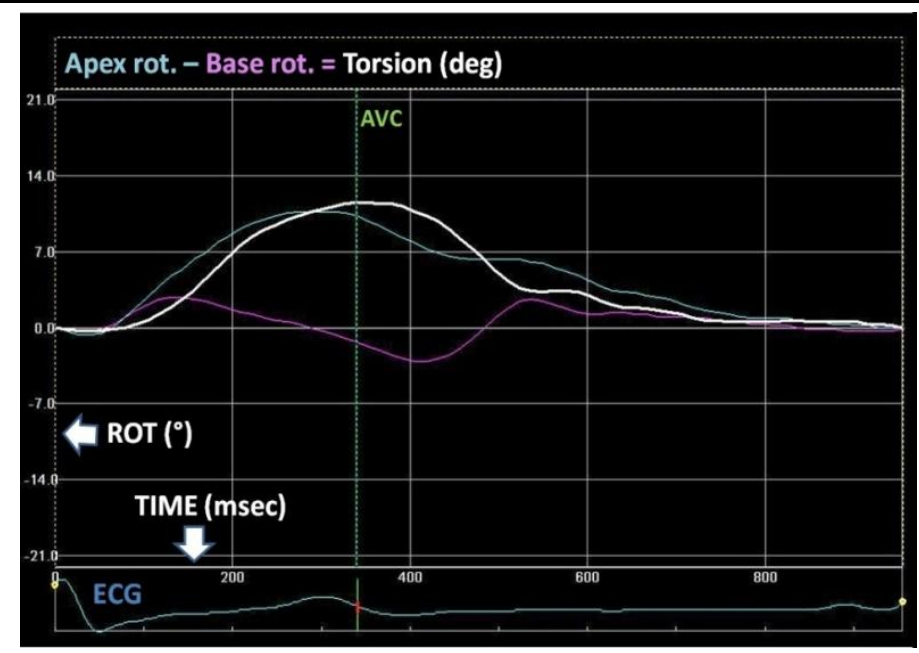

Fig. 1. Peak left ventricular twist and untwist curves in heart transplant patients.

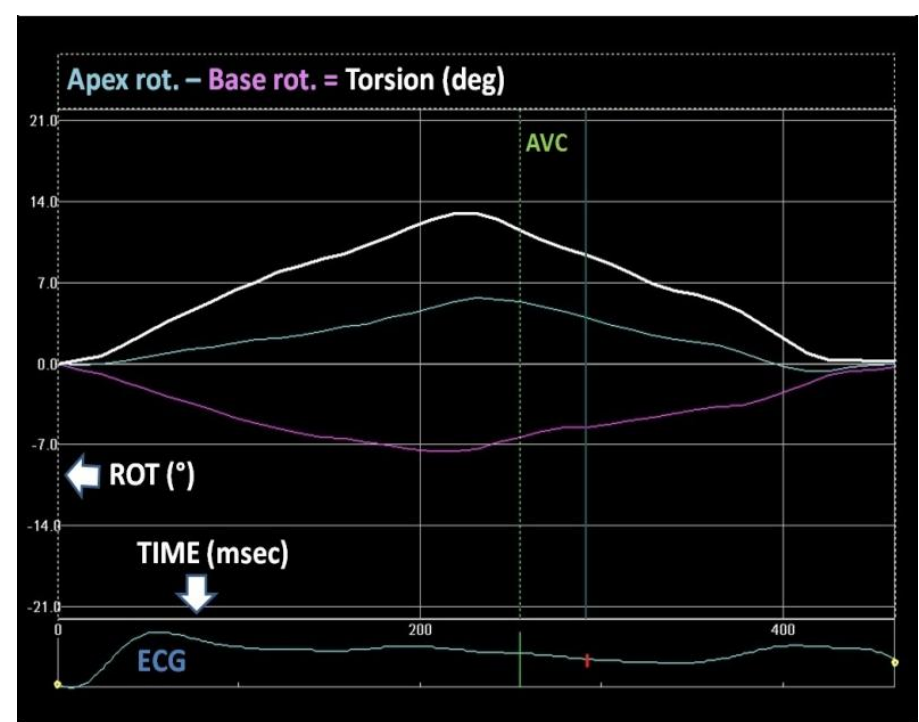

Fig. 2. Peak left ventricular twist and untwist curves in controls.

Finally, in HTX patients also rotation rate was significantly lower at basal level and preserved at apical level even if twist rate was significantly reduced (HTX Group: Basal Rotation Rate -51.6 \pm $20.01 \% \mathrm{sec}$, Apical Rotation Rate $69.55 \pm 25.7 \% \mathrm{sec}$, Twist Rate $128.3 \pm 12.4 \% \mathrm{sec}$; CTRL Group: Basal Rotation Rate $-75.23 \pm 15.2 \%$ sec, Apical Rotation Rate $64.7 \pm 15.7 \%$ sec, Twist Rate $137.6 \pm$ $14.3 \%$ sec; $p<0.0001, p$ NS, $p<0.005$, respectively).

LV twist was prolonged and onset of untwist (time to peak twist/systolic time) was delayed (HTX Group $100.5 \pm 11.09 \%$, CTRL Group $84.7 \pm 11.5 \%, p$ value $<0.0001)$.

Of interest a significant correlation was found between IVRT and untwist onset $(r=0.4581, p<0.001)$ (Fig.3).

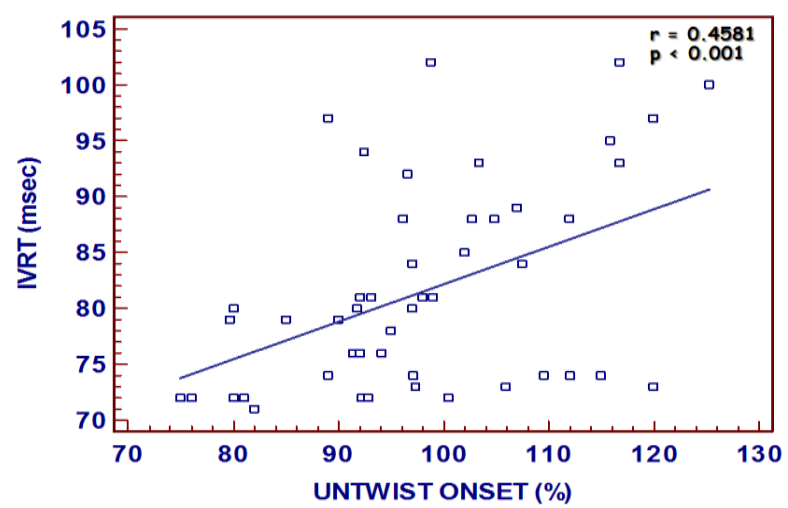

Fig. 3. Relationship between IVRT and untwisting onset in heart transplant patients. 
Significantly, although RV longitudinal strain and SR were impaired compared to controls, no significant correlations were found between RV and LV myocardial deformations ( all p NS).

In conclusion we divided HTX patients into two groups according to the median time after transplantation, that was 7 years, obtaining subgroups HTX1 (heart transplantation more than 7 years before) and HTX2 (heart transplantation less than 7 years before), and we did not observe any significant difference between them in all parameters considered (all p NS).

Intra- and interobserver variability were $3.2 \%$ and $4.8 \%$ for STE longitudinal, circumferential and radial values, $3.9 \%$ and $5.6 \%$ for $\mathrm{LV}$ twist, and $2.0 \%$ and $3.6 \%$ for untwisting onset.

\subsection{Main Findings}

To our knowledge this is the first study in which an integrated ventricular mechanics (longitudinal, circumferential and radial deformation, rotation, twist and untwist onset) in "healthy" heart transplant patients is performed.

The main finding of our study is that "healthy" (EF $>50 \%$, NYHA class I) HTX patients show impaired global biventricular myocardial deformation with a lesser degree of alteration or preservation of LV apical segments. In addition, the higher apical rotation may explain the maintenance of a normal amplitude of LV twist and thus ejection fraction. Finally, the mildly prolonged twist with consequent untwisting delay could play a major role in the onset of the early diastolic dysfunction.

\subsection{Conventional Assessment of Allograft Function}

We considered patients at least one year after heart transplantation to overcome the temporary allograft dysfunction that is a common finding early after the surgical intervention [25]. Indeed, Lunze et al [25] studied 44 young rejection-free HTX recipients during the first year after heart transplant and found a biventricular dysfunction (systolic and diastolic) in the early phases with a normalization of LV function after the first year post-transplant. On the other hand they found that RV systolic function remains abnormal 1 year-post-transplant.

Accordingly our "healthy" HTX patients showed a normal LVEF. However, a subtle diastolic alteration, revealed by a prolongation of IVRT, was also detectable; in addition, although its accuracy is still controversial, a normal $\mathrm{E} / \mathrm{E}$ ' ratio, suggestive of normal mitral filling pressures was shown. Likewise, also our study group showed right ventricular dysfunction, as confirmed by the reduction of TAPSE and systolic peak velocity of the PW tissue Doppler (S') as well.

In HTX patients RV impairment is a common finding but its frequency, causes, and naturalhistory are still undefined. Indeed, owing to lack of standardized objective criteria, the reported prevalence of RV dysfunction in HTX patients is highly variable: Klima et al reported 5.9\% of severe RV dysfunction using very restrictive criteria [26] and Campana et al observed $11.7 \%$ of increased right atrial pressure [27]; on the other hand Mastouri et al [28] demonstrated that 100\% of his HTX study group had RV impairment defined as TAPSE > 2 SD below normal values.

\subsection{Integrated Ventricular Mechanics}

Our HTX patients, although clinically "healthy", were characterized by a diffused reduction in LV systolic longitudinal, circumferential and radial, and RV systolic deformations (strain and strain rate).

Accordingly recent studies demonstrated a significant reduction in LV longitudinal deformations [1315]. Particularly, Pichler et al [15] observed that the reduction in longitudinal strain in "healthy" HTX patients does not deteriorate 3 years after the initial assessment and remains stable over the years as long as the LVEF is preserved; additionally our HTX patients did not show any difference in terms of STE data when comparing patients more or less than 7 years from heart transplantation.

Unlikely Saleh et al [13] found normal values of circumferential strain in HTX patients 1 year after transplantation, but he did not perform an integrated assessment of myocardial deformations limiting his analysis to longitudinal and circumferential deformation.

Of interest, in our patients we found different LV myocardial deformation values between LV apical and basal level. Indeed, the basal segments showed a homogeneous reduction of strain and SR indexes while at the apical level we found a lesser lowering of strain with preserved SR values. The different 
behavior of apical Strain and SR could reflect the relatively load independence of SR, which is more comparable to intrinsic contractility. [29]

This LV base-apex gradient was also confirmed by rotation data: compared to control group HTX patients showed a significantly lowered basal rotation with preserved apical rotation. As a consequence LV twist amplitude, which is the net difference in clockwise and counterclockwise rotation of the LV apex and base, was normal.

LV twist plays a major role in LV mechanics and ejection fraction maintenance and a significant correlation between LV torsion and EF has been shown [30]. Accordingly, our finding could explain the preservation of EF in healthy HTX patients, despite the reduction of longitudinal, circumferential and radial deformation.

Impairment of LV filling is also supported by our STE data, showing a mildly delayed untwisting. Indeed, as subsequent recoil of twist deformation ("untwist") contributes to LV diastolic relaxation and early diastolic filling, a delay of this recoil mechanism induces impairment of LV relaxation [3033].

Indeed Burns et al [34] showed that the untwisting delay significantly correlated to the invasive indexes of LV relaxation (prolonged $\tau$ ) but not to LV stiffness. Accordingly, we found a significant correlation between untwisting delay and IVRT, as shown in our previous study on cardiomyopathies, too [35].

\subsection{New Pathophysiological Insights}

Our "healthy" HTX pts showed, even in presence of a normal peak LV twist, a decreased LV twist rate (i.e. an increased duration of systolic twist) associated to concomitant LV and RV alterations of the regional myocardial deformation (decreased LV longitudinal, radial, circumferential strain and SR, basal rotation, and RV longitudinal strain and SR).

Potentially, several factors, could be taken into account to explain the subclinical impairment of both LV and RV: pretransplantation ischemia, brain death in the organ donor (myocardial injury determined by catecholamine surge after the sudden increase in intracranial pressure as a consequence of an intracranial hemorrhage or head trauma) [36], aftereffects of any open heart surgery [37], heart denervation.

On the other hand other causes may have a deeper impact on RV mechanics than LV: altered atrial architecture following heart transplantation, expecially after biatrial surgical technique, and elevated precapillary pulmonary resistance in the recipient's circulation.

Although ventricular functional interdependence was previously well defined [38, 39], the lack of correlation between LV and RV myocardial deformation properties in HTX patients probably reflects these different pathophysiological substrates.

Of interest a clear LV base-apex gradient is detectable and this apical compensatory mechanism is a mainstay to preserve the degree of LV systolic rotation and thus ensure a normal EF.

In presence of the above-mentioned pathophysiological substrates we speculated that regional alterations in LV mechanics following heart transplantation may reflect differences in terms of systolic wall stress. Indeed, according to the Laplace law, wall stress (afterload), owing to a smaller cavity diameter, is lower at the apex than at the base. These regional loading conditions may explain the preservation of apical myocardial function as a compensatory mechanism to the basal alterations.

\subsection{Study Limitations}

Although $70 \%$ of them was in ace-inhibitor therapy due to corticosteroids-related hypertension, our study population, according to inclusion criteria, had normal blood pressure values.

LV strain and SR indexes might be influenced by several factor, such as blood pressure, LV geometry and filling pressures; however, in our HTX study these parameters were comparable to the control group making the impact of such factors not relevant.

On the other hand the higher heart rate in our HTX group due to cardiac denervation could affect some functional data. Nevertheless, the untwist onset was corrected for heart rate by converting systolic interval to $100 \%$ as previously described [35,40]. Furthermore, the deformation parameters (longitudinal, radial and circumferential strain, twist) are relatively heart rate independent [41-42]. 


\section{CONCLUSIONS}

"Healthy" HTX patients, even in presence of impairment of global (longitudinal, circumferential, radial) myocardial deformation, show normal values of LV apical SR and rotation, which account for normal amplitude of LV twist and in turn global ejection fraction.

In addition, probably due to regional differences in wall stress, a base-apex gradient of these subclinical systolic dysfunction is detectable. Finally a delayed untwisting due to prolonged twist could be responsible of an early impaired LV diastolic filling.

Further studies are needed to assess the potential impact of these new pathophysiological insights on the management of the "healthy" heart transplant patients.

\section{ACKNOWLEDGEMENTS}

We have not conflict of interest to declare.

We give the permission to reproduce published material in all forms of media.

\section{REFERENCES}

[1]. Taylor DO, Stehlik J, Edwards LB, et al. Registry of the International Society for Heart and Lung Transplantation: twenty-sixth official adult heart transplant report-2009. J Heart Lung Transplant. 2009;28:1007-22.

[2]. Syeda B, Roedler S, Schukro C, et al. Transplant coronary artery disease: Incidence, progression and interventional revascularization. Int J Cardiol. 2005;104:269-74.

[3]. Edvardsen T, Gerber BL, Garot J, et al. Quantitative assessment of intrinsic regional myocardial deformation by Doppler strain rate echocardiography in humans: validation against threedimensional tagged magnetic resonance imaging. Circulation. 2002;106:50-6.

[4]. Leitman M, Lysyansky P, Sidenko S, et al. Two-dimensional strain: a novel software for realtime quantitative echocardiographic assessment of myocardial function. J Am Soc Echocardiogr. 2004;17:1021-9.

[5]. Pirat B, Khoury DS, Hartley CJ, et al. A novel feature-tracking echocardiographic method for the quantitation of regional myocardial function: validation in an animal model of ischemiareperfusion. J Am Coll Cardiol. 2008;51:651-9.

[6]. Notomi Y, Lysyansky P, Setser RM, et al. Measurement of ventricular torsion by twodimensional ultrasound speckle tracking imaging. J Am Coll Cardiol. 2005;45:2034-41.

[7]. Helle-Valle T, Crosby J, Edvardsen T, et al. New noninvasive method for assessment of left ventricular rotation: speckle tracking echocardiography. Circulation. 2005; 112:3149-56.

[8]. Kim HK, Sohn DW, Lee SE, et al. Assessment of left ventricular rotation and torsion with two dimensional speckle tracking echocardiography. J Am Soc Echocardiogr. 2007;20: 45-53.

[9]. Notomi Y, Setser RM, Shiota T, et al. Assessment of left ventricular torsional deformation by Doppler tissue imaging: validation study with tagged magnetic resonance imaging. Circulation. 2005;111:1141-7.

[10].Sarvari SI, Gjesdal O, Gude E, et al. Early postoperative left ventricular function by echocardiographic strain is a predictor of 1-year mortality in heart transplant recipients. J Am Soc Echocardiogr. 2012; 25(9):1007-14.

[11]. Kato TS, Oda N, Hashimura K, et al. Strain rate imaging would predict sub-clinical acute rejection in heart transplant recipients. Eur J Cardiothorac Surg. 2010;37:1104-10.

[12]. Sato T, Kato TS, Kamamura K, et al. Utility of left ventricular systolic torsion derived from 2dimensional speckle-tracking echocardiography in monitoring acute cellular rejection in heart transplant recipients. J Heart Lung Transplant. 2011;30:536-43.

[13]. Saleh HK, Villarraga HR, Kane GC, et al. Normal left ventricular mechanical function and synchrony values by speckle-tracking echocardiography in the transplanted heart with normal ejection fraction. J Heart Lung Transplant. 2011; 30:652-58

[14]. Syeda B, Höfer P, Pichler P, et al. Two-dimensional speckle tracking echocardiography in longterm heart transplant patients: a study comparing deformation parameters and ejection fraction 
derived from echocardiography and multislice computed tomography. Eur $J$ Echocardiogr. 2011; 12:490-496.

[15].Pichler P, Binder T, Höfer P, et al. Two-dimensional speckle tracking echocardiography in heart transplant patients: three-year follow-up of deformation parameters and ejection fraction derived from transthoracic echocardiography. Eur Heart J Cardiovasc Imaging. 2012;13:181-6.

[16]. Stewart S, Winters GL, Fishbein MC, et al. Revision of the 1990 working formulation for the standardization of nomenclature in the diagnosis of heart rejection. J Heart Lung Transplant 2005;24:1710-20.

[17].Sahn DJ, DeMaria A, Kisslo J, et al. Recommendations regarding quantification in M-mode echocardiography: results of a survey of echocardiographic measurements. Circulation. 1978;58:1072-83.

[18].Lester SJ, Ryan EW, Schiller NB, et al. Best method in clinical practice and research studies to determine left atrial size. Am J Cardiol. 1999; 84:829 - 832.

[19]. Schiller N, Shah PM, Crawford M, et al. Recommendations for quantification of the left ventricle by two-dimensional echocardiography: American Society of Echocardiography Subcommittee on Standards. J Am Soc Echocardiogr. 1989;2:358-68.

[20]. Nagueh S, Appleton CP, Gillebert TC, et al. Recommandations of the evaluation of diastolic dysfunctions by echocardiography. J Am Soc Echocardiogr. 2009;22:108 -128.

[21]. Nagueh SF, Middleton KJ, Kopelen HA, et al. Doppler tissue imaging: a noninvasive technique for evaluation of left ventricular relaxation and estimation of filling pressures. J Am Coll Cardiol. 1997; 30: 1527-1533

[22]. Kaul S, Tei C, Hopkins JM, Shah PM. Assessment of right ventricular function using twodimensional echocardiography. Am Heart J 1984;107:526-531.

[23]. Tuller D, Steiner M, Wahl A, et al. Systolic right ventricular function assessment by pulsed wave tissue Doppler imaging of the tricuspid annulus. Swiss Med Wkly 2005;135:461-468.

[24].Notomi Y, Martin-Miklovic MG, Oryszak SJ, et al. Enhanced ventricular untwisting during exercise: a mechanistic manifestation of elastic recoil described by Doppler tissue imaging. Circulation. 2006; 113:2524-33.

[25].Lunze FI, Colan SD, Gauvreau K, et al. Cardiac allograft function during the first year after transplantation in rejection-free children and young adults. Circ Cardiovasc Imaging. 2012;5:756-64.

[26]. Klima U, Ringes-Lichtenberg S, Warnecke G, et al. Severe right heart failure after heart transplantation. A singlecenter experience. Transpl Int 2005;18(3):326-332.

[27]. Campana C, Gavazzi A, Marioni R, et al: Right ventricular failure after heart transplantation: relationship with preoperative haemodynamic parameters. Transpl Int 1992;5 (Suppl 1):S221223.

[28]. Mastouri R, Batres Y, Lenet A, et al. Frequency, time course, and possible causes of right ventricular systolic dysfunction after cardiac transplantation: a single center experience. Echocardiography. 2013; 30:9-16.

[29]. Caiani EG, Weinert L, Lang RM, et al. The role of echocardiography in the assessment of cardiac function in weightlessness-Our experience during parabolic flights. Respir Physiol Neurobiol. 2009;169 Suppl 1:S6-9.

[30]. Sengupta PP, Tajik AJ, Chandrasekaran K, et al. Twist mechanics of the left ventricle. J Am Coll Cardiol. 2008;1(3):366-76.

[31]. Rademakers FE, Buchalter MB, Rogers WJ, et al. Dissociation between left ventricular untwisting and filling. Accentuation by catecholamines. Circulation. 1992;85:1572-81.

[32].Lester SJ, Tajik AJ, Nishimura RA, et al. Unlocking the mysteries of diastolic function deciphering the Rosetta Stone 10 years later. J Am Coll Cardiol. 2008;51:679-89.

[33].Notomi Y, Martin-Miklovic MG, Oryszak SJ, et al. Enhanced ventricular untwisting during exercise: a mechanistic manifestation of elastic recoil described by Doppler tissue imaging. Circulation. 2006; 113:2524-33.

[34]. Burns AT, La Gerche A, Prior DL, et al. Left ventricular untwisting is an important determinant of early diastolic function. $J$ Am Coll Cardiol Img 2009;2:709-16. 
[35].Pacileo G, Baldini L, Limongelli G, et al. Prolonged left ventricular twist in cardiomyopathies: a potential link between systolic and diastolic dysfunction. Eur J Echocardiogr. 2011;12(11):8419.

[36].Bittner HB, Chen EP, Biswas SS, et al. Right ventricular dysfunction after cardiac transplantation: Primarily related to status of donor heart. Ann Thorac Surg 1999;68 (5):16051611 .

[37].Lindstrom L, Wigstrom L, Dahlin LG, et al. Lack of effect of synthetic pericardial substitute on right ventricular function after coronary artery bypass surgery. An echocardiographic and magnetic resonance imaging study. Scand Cardiovasc J 2000;34(3):331-338.

[38]. Clyne CA, Alpert JS, Benotti JR. Interdependence of the left and right ventricles in health and disease. Am Heart J. 1989; 117(6):1366-73.

[39]. Santamore WP, Dell'Italia LJ. Ventricular interdependence: significant left ventricular contributions to right ventricular systolic function. Prog Cardiovasc Dis. 1998; 40(4):289-308.

[40]. Notomi Y, Martin-Miklovic MG, Oryszak SJ, et al. Enhanced ventricular untwisting during exercise: a mechanistic manifestation of elastic recoil described by Doppler tissue imaging. Circulation. 2006; 113:2524-33.

[41]. Suzuki R, Matsumoto $\mathrm{H}$, Teshima $\mathrm{T}$, et al. Influence of heart rate on myocardial function using two-dimensional speckle-tracking echocardiography in healthy dogs. J Vet Cardiol. 2013; 15:139-46.

[42]. Klitsie LM, Roest AA, van der Hulst AE, et al. Assessment of Intraventricular Time Differences in Healthy Children Using Two-Dimensional Speckle-Tracking Echocardiography. J Am Soc Echocardiogr. 2013; 26:629-39. 